\title{
Diagnosis and management of the epilepsies in adults and children: summary of updated NICE guidance
}

In this article by Vanessa Delgado Nunes and colleagues an editing error occurred in the section "Adjunctive treatment for generalised tonic-colonic seizures" (BMJ 2012;344:e281, doi:10. 1136/bmj.e281). The second bullet point should read: "If there are absence seizures or myoclonic seizures [not "If myoclonic seizures are absent or if juvenile myoclonic epilepsy is suspected"], do not offer carbamazepine, gabapentin, oxcarbazepine, phenytoin, pregabalin, tiagabine, or vigabatrin. (New recommendation.)"

Cite this as: BMJ 2012;344:e1173

๑ BMJ Publishing Group Ltd 2012 